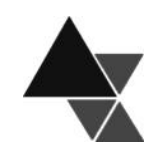

\title{
Perfil dos voluntários de universidade brasileira a respeito do consumo de pescado ${ }^{1}$
}

\author{
Erika da Silva Maciel ${ }^{2}$, Júlia Santos Vasconcelos ${ }^{3}$, Jaqueline Girnos Sonati ${ }^{4}$, \\ Luciana Kimie Savay-da-Silva ${ }^{5}$, Juliana Antunes Galvão ${ }^{6}$, Marília Oetterer $^{7}$
}

O objetivo deste trabalho foi descrever as características dos consumidores e não consumidores de pescado tendo por base uma amostra de integrantes de comunidade universitária paulista. O instrumento utilizado foi um questionário on line, constituído de itens relacionados ao consumo de pescado e principais características envolvidas nesse processo. Em conjunto foi utilizado o questionário International Physical Activity Questionaire, para avaliação do Nível de Atividade Física e informações antropométricas (peso e altura) autorreferidas. A amostra foi composta por 1966 voluntários da Universidade de São Paulo (Brasil). Quanto ao Nível de Atividade Física dos indivíduos que não consomem pescado observou-se que 29,2\% não atenderam a recomendação mínima de atividade física semanal, grupo que agrega os sedentários e os indivíduos insuficientemente ativos A e B. Foi observado que $22 \%$ apresentaram estado nutricional classificado como sobrepeso ou obesidade grau I. A maioria dos voluntários $(27,11 \%)$ apresentou frequência de consumo de pescado de uma vez por semana. Do total, 5,13\% não consumiram o pescado, destacando-se motivos como: sabor não atraente; falta de hábito; problemas de saúde (alergias); não saber preparar; dificuldade de avaliar o produto pela aparência; e indisponibilidade do produto no local de compra habitual. Fatores como preço e qualidade também se mostraram interferentes na decisão dos voluntários quanto ao consumir ou não o produto.

Palavras-chave: comunidade universitária, consumo de pescado, estilo de vida.

\section{Characterization of the profile of volunteers from a Brazilian university on fish consumption}

The objective of this study was to describe the characteristics of fish consumers and people who do not consume fish in a sample of the university community in São Paulo. An on line questionnaire consisting of items related to fish consumption and main features involved in this process was used. Also, we used the International Physical Activity Questionnaire to assess the level of physical activity and gather information anthropometric (height and weight) self-reported. The sample consisted of 1966 volunteers from the University of São Paulo (Brasil). Results show that 29.2\%, regarding the physical activity level, of the volunteers were insufficiently active

\footnotetext{
${ }^{1}$ Financiamento FAPESP: Processos 2008/09941-4.

${ }^{2}$ Doutora Universidade de São Paulo (USP), Campus “Luiz de Queiroz”, Piracicaba, SP. Correspondência: Avenida Pádia Dias 11, Bairro Agronomia, CEP 13418-900. Piracicaba, SP. Fone/fax: 55 (19) 34294150. Ramal: 36. E-mail: erikasmaciel@gmail.com.

${ }^{3}$ Graduanda em Ciência dos Alimentos, Escola Superior de Agricultura "Luiz de Queiroz" (ESALQ), USP.

${ }^{4}$ Doutora, Faculdade de Educação Física da Universidade Estadual de Campinas (UNICAMP).

${ }^{5}$ Mestre, Departamento de Agroindústria, Alimentos e Nutrição, ESALQ (USP).

${ }^{6}$ Doutora, Departamento de Agroindústria, Alimentos e Nutrição, ESALQ (USP)

${ }^{7}$ Profa. Titular, Departamento de Agroindústria, Alimentos e Nutrição, ESALQ (USP).
} 
individuals A and B and sedentary, in the group that does not consume fish. In terms of nutritional status, $22 \%$ is overweight or has obesity level 1. Most volunteers (27.11\%) consume fish once a week. Of the total, $5.13 \%$ does not consume fish, mainly because of the unattractive taste; lack of habit and health problems (allergies); do not know how to prepare; difficulty to evaluate the product for appearance; and because the product is not available at the usual place of purchase. Factors such as price and quality did not affect the decision-making process for volunteers to purchase the product.

Key-words: university community, fish consumption, lifestyle.

\section{INTRODUÇÃO}

O consumo de pescado pode ser influenciado por diversos fatores, entre esses se destacam os socioeconômicos, os padrões de consumo alimentar, suas características pessoais, estado de saúde e suas dimensões atitudinais [1]. Nos últimos anos houve um aumento de pesquisas com objetivo de avaliar o potencial benefício à saúde, associado ao consumo de pescado, inclusive na proteção contra algumas doenças crônicas. Entretanto, os resultados têm sido inconsistentes devido a dificuldade para avaliar o consumo de pescado e de seus produtos [2].

O aumento de interesse pelo pescado nos últimos anos está vinculado às informações quanto ao seu valor nutricional e sua associação com melhorias para a saúde, observadas em populações que possuem esse alimento como base da alimentação. Entre os benefícios, foram identificados declínios dos níveis de colesterol, da incidência de acidente vascular cerebral, de doença cardíaca, e, possivelmente o risco de Alzheimer. Este alimento pode aumentar a função cognitiva em adultos e impedir o nascimento de crianças com baixo peso e o parto pré-termo [3].

O pescado apresenta balanço protéico, de vitaminas, principalmente as do complexo $\mathrm{B}$, vitamina A e D; minerais como sódio, ferro, potássio, magnésio, cobre, enxofre, fósforo e iodo; além de baixo valor calórico [4]. A ingestão regular de pescado tem sido associada à redução dos níveis de triglicerídeos, na manutenção da pressão sanguínea, no mecanismo de coagulação e ritmo cardíaco, na prevenção do câncer (mama, próstata e cólon) e na redução da incidência de aterosclerose [5].

Apesar dos diversos benefícios decorrentes da ingestão de pescado, o seu consumo ainda é baixo em muitos países. Tal fato pode estar vinculado aos aspectos culturais [6], preço elevado e problemas na cadeia de produção ${ }^{[7]}$.

Face ao exposto, o trabalho teve como objetivo principal a descrição das características dos consumidores e não consumidores de pescado integrantes de amostra de comunidade universitária paulista.

\section{MATERIAL E MÉTODOS}

Os participantes da pesquisa constituem uma amostra $(n=1966)$ não probabilística de conveniência, da comunidade da Universidade de São Paulo - USP e contou com a participação voluntária de alunos e servidores dos Campi de São Paulo, Piracicaba, Pirassununga, São Carlos, Ribeirão Preto, Lorena e Bauru.

$\mathrm{O}$ instrumento utilizado foi previamente testado e validado, constituído de itens relacionados ao consumo e principais características envolvidas nesse processo. Em conjunto foi utilizado o questionário International Physical Activity Questionaire (IPAQ) versão 8, forma curta e semana normal, para avaliação do nível de atividade física e informações antropométricas (peso e altura) autorreferidas.

As informações sobre a pesquisa, bem como os instrumentos, foram hospedadas no site do Grupo de Estudo e Extensão de Inovação Tecnológica e Qualidade do Pescado (GETEP), da Escola Superior de Agricultura Luiz de Queiroz (ESALQ) - USP. Para tornar possível o acesso aos mesmos foi enviado convite via e-mail aos membros da comunidade, sendo utilizadas também outras fontes de divulgação como redes sociais, mural informativo e palestras.

A coleta de dados ocorreu durante o período de março de 2010 a agosto de 2010. A identificação de 
usuário de e-mail e número USP foi utilizada para garantir que somente a comunidade universitária da USP pudesse acessar os instrumentos. O sistema foi construído de forma a permitir interrupção de preenchimento dos formulários em determinado momento e a continuação a posteriori. Também foi permitido desistir do preenchimento a qualquer momento, bastando para isso não concluir a remessa das informações. Dessa forma, a amostra foi composta por 1966 voluntários, alunos de graduação e pós-graduação, servidores docentes e não docentes da USP oriundos dos sete Campi.

As análises dos dados do IPAQ foram elaboradas seguindo a metodologia proposta pela Organização Mundial de Saúde - OMS. O IPAQ foi desenvolvido pela OMS, validado, avaliado e utilizado no Brasil e em outros países [8,9]. Esse instrumento é composto por 8 perguntas relacionadas à frequência e duração da realização de atividades físicas moderadas, vigorosas e de caminhada tendo como referência a última semana ou uma semana típica.

Para classificação dos níveis de atividade física foram utilizados os critérios descritos por Matsudo et al. ${ }^{[8]}$ que classificam cada um dos indivíduos de acordo com as atividades que os mesmos exercem ao longo dos dias da semana. Tais atividades podem ser "vigorosas", quando demandam um grande esforço físico e que fazem respirar muito mais forte que o normal ou, atividades "moderadas", que requerem algum esforço físico e produzem uma respiração um pouco mais forte que o normal. Os dados são fornecidos com base na última semana e permitem a classificação do indivíduo conforme descrito a seguir:

Sedentário - aquele indivíduo que não realizou atividade física durante pelo menos 10 minutos contínuos ao longo da semana.

Insuficientemente ativo - aquele indivíduo que realiza atividade física com duração mínima de 10 minutos por semana, porém insuficiente para ser classificado como ativo. Esses indivíduos ainda podem ser classificados em dois grupos, a saber: grupo formado pelos indivíduos que atingem pelo menos um dos critérios da recomendação para a frequência (5 dias por semana) ou duração (150 minutos por semana); grupo composto por indivíduos que não atende nenhum dos critérios recomendados.
Ativo - aquele que cumpre as recomendações de atividades vigorosas (maior ou igual a 3 dias por semana e maior ou igual a 20 minutos por sessão) e/ou atividade moderada ou caminhada (maior ou igual a 5 dias por semana e pelo menos 30 minutos por sessão) e/ou qualquer atividade somada maior ou igual a 5 dias por semana e pelo menos 150 minutos por semana.

Muito ativo - aquele indivíduo que cumpre as recomendações de atividades vigorosas (maior ou igual a 5 dias por semana e 30 minutos por sessão) ou atividades vigorosas (pelo menos 3 dias por semana, com 20 minutos por sessão, acrescidos de uma atividade moderada ou caminhada que deve ser de pelo menos 5 dias por semana e 30 minutos por sessão).

Foi elaborada análise descritiva dos resultados, considerando o grupo de consumidores e dos não consumidores de pescado, análise de frequência e correlação de Spearman envolvendo idade e frequência do consumo de pescado. As análises foram realizadas adotando-se os recursos do software SPSS 15.0.

A pesquisa foi aprovada pelo Comitê de Ética em Pesquisa da Escola Superior de Agricultura "Luiz de Queiroz" - ESALQ, Universidade de São Paulo (USP), sob o protocolo de número 21 (COET/046).

\section{RESULTADOS E DISCUSSÃO}

Nesta pesquisa, observa-se que (Tabela 1) a maioria é representada pelos solteiros $(65,9 \%)$, mulheres $(61,6 \%)$ e alunos de graduação $(52,6 \%)$ oriundos do Campus de São Paulo (50,9\%).

A média geral para idade foi de 30,42 anos $( \pm 12,41)$ e a maior média foi identificada entre os participantes do Campus de Bauru, 42,27 anos $( \pm 11,98)$. Os voluntários dos Campi de Lorena e Piracicaba apresentaram as menores médias de idade, 29,08 anos $( \pm 12,65)$ e 29,23 anos $( \pm 11,70)$, respectivamente. No Campus de São Paulo a média (da idade) foi de 30,21 anos ( $\pm 12,67)$, no de Pirassununga e Ribeirão Preto, 31,67 anos $( \pm 11,53)$ e 31,70 anos $( \pm 12,26)$, respectivamente. Em São Carlos, a média de idade alcançou 32,91 anos ( $\pm 13,05)$. 
Tabela 1. Perfil da amostra de participantes da pesquisa em comunidade universitária, 2011.

\begin{tabular}{lrc}
\hline \multicolumn{1}{c}{ Variável } & $\boldsymbol{n}$ & Frequência (\%) \\
\hline Estado civil & 1295 & 65,9 \\
Solteiro & 455 & 23,1 \\
Casado & 139 & 7,1 \\
Vivendo como casado & 69 & 3,5 \\
Separado/Divorciado & 8 & 0,4 \\
Viúvo & 1966 & 100,0 \\
\hline Total & & \\
\hline Categoria & 1035 & 52,6 \\
Aluno de graduação & 347 & 17,7 \\
Aluno de pós-graduação & 303 & 15,4 \\
Servidor não docente & 248 & 12,6 \\
Servidor docente & 33 & 1,7 \\
Outros & 1966 & 100,0 \\
\hline Total & & \\
\hline Campi & 1000 & 50,9 \\
São Paulo & 482 & 24,5 \\
Piracicaba & 240 & 12,2 \\
Ribeirão Preto & 84 & 4,3 \\
Pirassununga & 62 & 3,2 \\
Lorena & 59 & 3,0 \\
São Carlos & 23 & 1,2 \\
Bauru & 16 & 0,8 \\
Outros* & 1966 & 100,0 \\
\hline Total & &
\end{tabular}

* Demais centros de pesquisa localizados fora dos Campi da USP.

Considerando a totalidade dos participantes, a avaliação da frequência de consumo de pescado (Tabela 2) demonstra que apenas 17,39\% em Bauru; 11,4\% em São Paulo; 8,33\% Pirassununga; 7,91\% em Ribeirão Preto; 7,67\% em Piracicaba; 6,77\%; em São Carlos; e 1,61\% em Lorena, consumiram pescado de acordo com a frequência preconizada (duas vezes ou mais na semana) pela Food and Agriculture Organization (FAO) ${ }^{[10]}$.

Do total de 1966 voluntários, apenas 101 $(5,13 \%)$ não consumiram pescado. Dentre os motivos que levaram as pessoas a não consumir o pescado destacam-se o sabor não atraente $(53,4 \%)$; a falta de hábito (35,6\%); problemas de saúde (alergias de algumas espécies de pescado) que impossibilitam o consumo (6,86\%); não saber preparar $(1,96 \%)$, dificuldade de avaliar o produto pela aparência $(0,98 \%)$ e indisponibilidade do produto no local habitual de compra $(0,98 \%)$.
Estudo envolvendo comunidades costeiras em Macau - Rio Grande do Norte, evidenciou a importância dos aspectos socioculturais e dos hábitos alimentares envolvidos no consumo do pescado e a relevância do desenvolvimento de ações visando a otimização e o aproveitamento integral do pescado [11].

A falta de hábito de consumo de pescado no Brasil tem sido demonstrada por meio de estudos envolvendo crianças ainda na fase escolar. Pesquisa realizada entre escolares do município de Piracicaba identificou que $43 \%$ deles indicaram o pescado como alimento de menor aceitação [12].

A Pesquisa de Orçamento Familiar (POF) 2002-2003 estimou a aquisição per capita de pescado em 4,6 kg/ano. Nas áreas rurais o consumo (per capita), foi de $9,9 \mathrm{~kg} /$ ano (maior que a média nacional), enquanto nos centros urbanos o consumo foi de 3,4 $\mathrm{kg} / \mathrm{ano}{ }^{[13]}$. 
Tabela 2. Frequência de consumo de pescado de comunidade universitária, de acordo com o Campus, 2011.

\begin{tabular}{|c|c|c|c|c|c|c|c|}
\hline \multirow[b]{2}{*}{ Campus } & \multicolumn{7}{|c|}{ Frequência } \\
\hline & 2 ou mais/semana & $1 \mathrm{x} /$ semana & 2 a 3x/mês & 1x/mês & Raramente & Nunca & Total \\
\hline \multirow{2}{*}{ Piracicaba } & 37 & 161 & 139 & 77 & 53 & 15 & 482 \\
\hline & $(7,67)$ & $(33,40)$ & $(28,83)$ & $(15,97)$ & $(10,99)$ & $(3,11)$ & {$[24,51]$} \\
\hline \multirow{2}{*}{ São Paulo } & 114 & 241 & 253 & 171 & 160 & 61 & 1000 \\
\hline & $(11,40)$ & $(24,10 \%)$ & $(25,30)$ & $(17,10)$ & $(16,00)$ & $(6,10)$ & {$[50,86]$} \\
\hline \multirow{2}{*}{ São Carlos } & 4 & 12 & 16 & 16 & 8 & 3 & 59 \\
\hline & $(6,77)$ & $(20,33)$ & $(27,11)$ & $(27,11)$ & $(13,55)$ & $(5,08)$ & {$[3,00]$} \\
\hline \multirow{2}{*}{ Ribeirão Preto } & 19 & 69 & 56 & 48 & 38 & 10 & 240 \\
\hline & $(7,91)$ & $(28,75)$ & $(23,33)$ & $(20,00)$ & $(15,83)$ & $(4,16)$ & {$[12,20]$} \\
\hline \multirow{2}{*}{ Pirassununga } & 7 & 29 & 23 & 14 & 9 & 2 & 84 \\
\hline & $(8,33)$ & $(34,52)$ & $(27,38)$ & $(16,66)$ & $(10,71)$ & $(2,38)$ & {$[4,27]$} \\
\hline \multirow{2}{*}{ Bauru } & 4 & 6 & 8 & 2 & 2 & 1 & 23 \\
\hline & $(17,39)$ & $(26,08)$ & $(34,78)$ & $(8,69)$ & $(8,69)$ & $(4,34)$ & {$[1,16]$} \\
\hline \multirow{2}{*}{ Lorena } & 1 & 10 & 14 & 12 & 19 & 6 & 62 \\
\hline & $(1,61)$ & $(16,12)$ & $(22,58)$ & $(19,35)$ & $(30,64)$ & $(9,67)$ & {$[3,15]$} \\
\hline \multirow{2}{*}{ Outros* } & 1 & 5 & 1 & 4 & 2 & 3 & 16 \\
\hline & $(6,25)$ & $(31,25)$ & $(6,25)$ & $(25,00)$ & $(12,5)$ & $(18,75)$ & {$[0,81]$} \\
\hline \multirow{2}{*}{ Total } & 187 & 533 & 510 & 344 & 291 & 101 & 1966 \\
\hline & {$[9,51]$} & {$[27,11]$} & {$[25,94]$} & {$[17,49]$} & {$[14,80]$} & {$[5,13]$} & {$[100]$} \\
\hline
\end{tabular}

A POF 2008-2009 mostrou que a aquisição de pescado foi de $4 \mathrm{~kg} /$ per capita ao ano. A Região Norte apresentou uma média de $17,5 \mathrm{~kg}$, quantidade que supera os valores observados nas demais regiões e a média nacional. Nas regiões Nordeste, Sudeste, Sul e Centro Oeste, os valores observados foram de 4,9; 2,0; 1,5 e $1,6 \mathrm{~kg} /$ ano, respectivamente ${ }^{[14]}$.

O consumo de pescado é considerado bastante heterogêneo no Brasil. Wiefels et al. [15] identificaram que em Aracajú o consumo (médio) de pescado era de $16,8 \mathrm{~kg} / \mathrm{pessoa} / \mathrm{ano}$, enquanto em Brasilia, 12,8 kg/pessoa/ano; em Recife, 8 $\mathrm{kg} /$ pessoa/ano, no Rio de Janeiro 16,4 kg/pessoa/ano e em São Paulo 15,3 kg/pessoa/ano.

Dados do Ministério da Pesca e Aquicultura [16] indicam que o consumo (aparente) de pescado per capita no Brasil aumentou de $6,4 \mathrm{~kg} / \mathrm{habitante} / \mathrm{ano}$, em 2003, para $9 \mathrm{~kg} /$ habitante/ano em 2009, o que representa um crescimento de $40 \%$ durante o período entre as duas pesquisas de orçamentos familiares. Em 2009, o volume total consumido pela população brasileira foi de 1,7 milhões de toneladas. Deste total, $69,4 \%$ foi produzido no Brasil ${ }^{[17]}$.
Em contrapartida, estudo para validação de questionário, visando aferir o consumo de pescado na Espanha, Islândia e Irlanda, envolvendo 60 indivíduos com idade entre 20 e 40 anos, identificou que a frequência do consumo foi mais elevada na Espanha, onde $70 \%$ dos participantes consomem pescado duas vezes ou mais por semana. As proporções de $47 \%$ e $10 \%$ foram verificadas, respectivamente, para a Islândia e Irlanda [18].

A ingestão per capita de pescado no Brasil, é relativamente baixa quando comparada ao consumo mundial ou ao consumo de outros tipos de carnes. O consumo de carne bovina atinge, por exemplo, 37,1 $\mathrm{kg} /$ ano no Brasil, enquanto que no mundo essa média é de 7,8 kg/ano. Para aves, a média nacional é de 31,2 $\mathrm{kg} / \mathrm{ano}$ e a mundial é de $7,4 \mathrm{~kg} / \mathrm{ano}{ }^{[19]}$. Ou seja, o Brasil consome cinco vezes mais carne bovina do que a média de consumo mundial e não atinge $50 \%$ da média mundial do consumo de pescado. Tal fato pode ser justificado pela alta produção nacional de gado. Estima-se que em 2008 essa produção totalizou 65 milhões de toneladas [20], enquanto que a produção de pescado atingiu, no mesmo ano, 1,17 milhões de toneladas ${ }^{[17]}$. 
Entretanto, quando se considera a comercialização nacional é notável o aumento de importações visando atender às demandas em períodos considerados especiais como é a "Semana Santa" e as festas de final de ano.

No Estado de São Paulo, o preço do pescado pode ser superior a duas vezes o valor médio da carne bovina de melhor qualidade: enquanto encontra-se carne bovina (filé mignon) por $\mathrm{R} \$ 28,24 \mathrm{~kg}$ [21], o pescado pode chegar a até $\mathrm{R} \$ 65,00 \mathrm{~kg}$ (camarão rosa - Penaeus brasiliensis) ${ }^{[22] .}$

Com isso, o consumo de pescado também sofre interferências pelo alto preço e pela ocorrência de problemas de controle de qualidade na cadeia de produção. Em relação ao pescado comercializado, os consumidores brasileiros destacam pouca variedade disponível no mercado, baixo rendimento do produto e dificuldades de se encontrar produto fresco e com aparência saudável [23].

Em relação ao perfil dos indivíduos que consomem pescado $(n=1865)$ observa-se (Tabela 3$)$ que há um predomínio dos indivíduos solteiros $(64,6 \%)$, mulheres $(61,7 \%)$, alunos de graduação (51,5\%), do Campus de São Paulo (50,3\%), com nível de atividade física predominantemente ativo $(51,0 \%)$ e classificados na faixa de normalidade de IMC $(62,8 \%)$. Note-se também que parcela considerável tenha sido classificada com sobrepeso $(25,2 \%)$ e obesidade I $(6,3 \%)$

Similarmente a esses resultados o perfil dos indivíduos que não consomem pescado $(n=101)$ (Tabela 4) apresenta predomínio dos solteiros (90\%), do sexo feminino $(62,6 \%)$, alunos de graduação (74,7\%), do Campus de São Paulo (60,4\%).

Em relação ao Nível de Atividade Física (NAF) destaca-se que, embora 61,6\% dos participantes que não consomem pescado tenham sido classificados como ativos, quando considerado o grupo dos indivíduos insuficientemente ativos A e B e os sedentários há um total de $29,2 \%$ dos indivíduos que não atendem a recomendação de atividade física semanal. Os resultados envolvendo o estado nutricional (com base no IMC) indicam que a maioria $(72,7 \%)$ dos sujeitos foi classificada no intervalo relativo à normalidade. Fora desse intervalo, foram identificados $(22,1 \%)$ os grupos de indivíduos com sobrepeso $(15,1 \%)$ e com obesidade grau I $(7 \%)$.
As mudanças dos hábitos alimentares têm sido outro fator importante quando se considera o consumo de pescado. A redução do consumo de frutas, hortaliças, leguminosas e pescado tem sido observada em diferentes países e há indícios de que as gerações mais novas têm adotado, com mais frequência, esse comportamento ${ }^{[24]}$.

A mudança no padrão alimentar, caracterizada pela melhoria das condições de vida nos países desenvolvidos, tem sido acompanhada pelo aumento da frequência de doenças nutricionais, sendo a mais importante delas a obesidade e suas consequências como as Doenças Crônicas Não Transmissíveis (DCNT). Essa transição nutricional, que passa da carência de nutrientes para o excesso, fez com que a obesidade virasse uma epidemia [25].

O padrão tradicional alimentar de populações do Mediterrâneo, por exemplo, oferece como base alimentar o pescado, entre outros alimentos, e há evidências consistentes das propriedades de promoção da saúde relacionadas a esse tipo de padrão [26,27].

Embora a discussão sobre esse modelo de dieta mediterrânea tenha se originado há alguns anos, somente no fim da última década os resultados das pesquisas epidemiológicas confirmaram o potencial, indicando o seu importante papel para a prevenção das DCNT, desde que associado a um estilo de vida ativo [28].

Todavia, em estudo transversal para avaliar a dieta e o estilo de vida de 210 estudantes universitários da Itália e Espanha, com idade média de 27 anos, foram observadas diferenças em relação ao consumo de alguns alimentos. O grupo composto pelos cereais foi consumido com maior frequência pelos estudantes italianos, enquanto que o pescado e legumes, por estudantes espanhóis. O excesso de peso foi maior entre os estudantes espanhóis, apesar de também apresentarem maior nível de atividade física, resultado que demostra a alteração no padrão alimentar deste país [24].

Fatores sociais como grupos de referência e família exercem grande influência no comportamento de compra do indivíduo, principalmente no que diz respeito aos produtos alimentícios. Reconhece-se que o padrão de consumo se inicia no âmbito da família, agente principal na construção dos valores do indivíduo [29]. 
Tabela 3. Perfil dos indivíduos de comunidade universitária que consomem pescado, 2011.

\begin{tabular}{|c|c|c|}
\hline Variável & $n$ & Frequência (\%) \\
\hline \multicolumn{3}{|l|}{ Estado civil } \\
\hline Solteiro & 1205 & 64,6 \\
\hline Casado & 449 & 24,1 \\
\hline Vivendo como casado & 136 & 7,3 \\
\hline Separado/Divorciado & 68 & 3,6 \\
\hline Viúvo & 7 & 0,4 \\
\hline \multicolumn{3}{|l|}{ Sexo } \\
\hline Masculino & 715 & 38,3 \\
\hline Feminino & 1150 & 61,7 \\
\hline \multicolumn{3}{|l|}{ Categoria } \\
\hline Aluno de graduação & 961 & 51,5 \\
\hline Aluno de pós-graduação & 333 & 17,9 \\
\hline Servidor não docente & 292 & 15,7 \\
\hline Servidor docente & 247 & 13,2 \\
\hline Outros & 32 & 1,7 \\
\hline \multicolumn{3}{|l|}{ Campi } \\
\hline Piracicaba & 467 & 25,0 \\
\hline São Paulo & 939 & 50,3 \\
\hline São Carlos & 56 & 3,0 \\
\hline Ribeirão Preto & 230 & 12,3 \\
\hline Pirassununga & 82 & 4,4 \\
\hline Bauru & 22 & 1,2 \\
\hline Lorena & 56 & 3,0 \\
\hline Outros & 13 & 0,7 \\
\hline \multicolumn{3}{|c|}{ Nível de Atividade Física (NAF) } \\
\hline Sedentário & 108 & 5,8 \\
\hline Insuficientemente ativo B & 400 & 21,4 \\
\hline Insuficientemente ativo A & 141 & 7,6 \\
\hline Ativo & 951 & 51,0 \\
\hline Muito ativo & 265 & 14,2 \\
\hline \multicolumn{3}{|l|}{ Índice de Massa Corporal } \\
\hline Baixo peso & 81 & 4,3 \\
\hline Normal & 1172 & 62,8 \\
\hline Sobrepeso & 470 & 25,2 \\
\hline Obesidade I & 118 & 6,3 \\
\hline Obesidade II & 20 & 1,1 \\
\hline Obesidade III & 4 & 0,2 \\
\hline
\end{tabular}


Tabela 4. Perfil dos indivíduos de comunidade universitária que não consomem pescado, 2011.

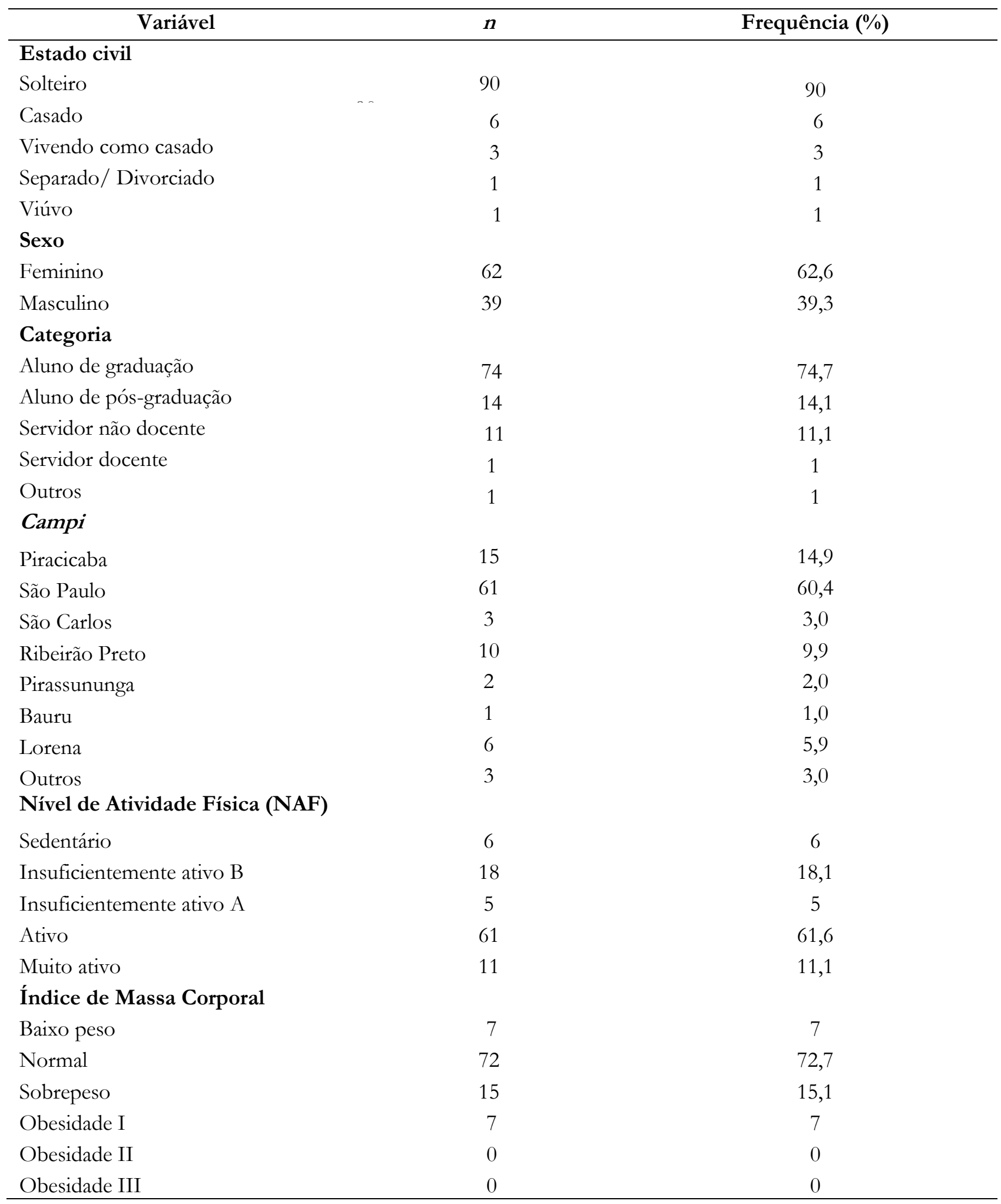


Os hábitos alimentares também são determinados pela idade dos consumidores. Essa variável parece ser um dos fatores pessoais mais discriminativos para a percepção da qualidade dos alimentos. Com as mudanças no perfil etário da população, verificadas mais intensamente em meados dos anos 90, com destaque para a diminuição das taxas de natalidade e o aumento da expectativa de vida, verificam-se alterações nos padrões de consumo e hábitos alimentares e, consequentemente, impactos em todos os setores da indústria alimentícia [30].

Neste estudo verificou-se que a idade (média em anos) dos indivíduos que consomem pescado duas ou mais vezes por semana foi maior $(35,36 \pm 7,82)$ do que aqueles indivíduos que não o consomem $(24,46$ $\pm 14,25)$. A análise de correlação de Spearman entre esses fatores demonstrou que há uma associação positiva e significante $(\phi=0,01)$ entre os mesmos, ou seja, quanto maior a idade do indivíduo maior é sua frequência de consumo de pescado.

Um dos fatores de destaque em relação ao consumo de pescado é o atual interesse das pessoas para a relação desse alimento com a saúde, longevidade e segurança do alimento [31]. Tal comportamento tem aumentado a demanda por produtos com características especiais como os orgânicos e funcionais.

São marcantes as diferenças socioeconômicas e culturais entre os consumidores dentro de um município, estado e país. Essas diferenças determinam os distintos níveis de percepção que cada grupo de consumidores tem em relação aos alimentos, em particular ao pescado. Os produtos de pescado são oriundos de centenas de espécies e apresentados de diferentes formas, tornando ainda mais complexa a percepção de qualidade que está sendo constantemente moldada pelo consumidor para cada um dos produtos de particular interesse [32].

Todavia, os consumidores estão mais exigentes em suas escolhas relativas ao consumo alimentar. Questões de qualidade dos alimentos se moveram para o primeiro plano das preocupações dos consumidores, estratégias da indústria e, em alguns casos, a política governamental. No Canadá, por exemplo, uma variedade de iniciativas de políticas públicas e do setor privado surgiu com o objetivo de reduzir a assimetria de informação dos consumidores em matéria de segurança e qualidade dos alimentos, em parte focada nos atributos da rastreabilidade [33].
Iniciativas como essas devem ser estimuladas, tendo em vista que o consumo de pescado está atrelado às questões de saúde e é o principal atributo que desperta o interesse de expressiva parcela dos consumidores ${ }^{[18]}$.

Sendo assim, embora o pescado apresente características favoráveis ao consumo, como o alto valor nutricional, também é um produto altamente perecível, e tal fato deve ser considerado por todos os atores da cadeia de produção e comercialização de modo que seja assegurada ao consumidor as características nutricionais, sensoriais e de qualidade.

\section{CONCLUSÃO}

Com base na amostra de voluntários foi possível caracterizar os consumidores de pescado como pessoas que se preocupam com os benefícios que este produto pode representar para a saúde. Notese também que os voluntários que revelaram baixo ou nulo consumo, o fazem, principalmente, devido a escassez de comercialização de produtos de conveniência, alto valor agregado e falta de garantia de qualidade do mesmo.

\section{AGRADECIMENTOS}

Ao Programa de Pós-graduação do Centro de Energia Nuclear na Agricultura (CENA).

Ao Conselho Nacional de Desenvolvimento Científico e Tecnológico (CNPq) pela bolsa doutorado concedida.

À Fundação de Amparo à Pesquisa do Estado de São Paulo (FAPESP) pelo financiamento.

\section{REFERÊNCIAS}

[1] Trondsen T, Scholderer J, Lund E, Eggen AE. Perceived barriers to consumption of fish among Norwegian women. Appetite. 2003;41(3):301-14.

[2] Mina K, Fritschi L, Knuiman M. A valid semiquantitative food frequency questionnaire to measure fish consumption. Eur J Clin Nutr. 2007;61(8):1023-31.

[3] Burger J. Fishing, fish consumption, and awareness about warnings in a university community in central New Jersey in 2007, and comparisons with 2004. Environ Res. 2008;108(1):107-16. 
[4] Piber Neto E. Enriquecimento do ovo: utilização de óleos de peixes e alga marinha como fontes de ácidos graxos poliinsaturados ômega-3 em rações de galinhas [dissertação]. São Paulo: Faculdade de Medicina Veterinária e Zootecnia, Universidade de São Paulo, 2006. 72 p.

[5] Souza PHM, Souza Neto MH, Maia GA. Componentes funcionais nos alimentos. Boletim da Sociedade Brasileira de Ciência e Tecnologia de Alimentos. 2003;37(2):127-135.

[6] Pieniak Z, Verbeke W, Scholderer J. Health-related beliefs and consumer knowledge as determinants of fish consumption. J Hum Nutr Diet. 2010;23(5):480-88.

[7] Oetterer M. Industrialização do pescado cultivado. Guaíba: Editora Agropecuária; 2002. 200 p.

[8] Matsudo SM, Matsudo VR, Araújo T, Andrade D, Andrade E, Oliveira L, et al. Nível de atividade física na população do Estado de São Paulo: análise de acordo com o gênero, idade e nível sócio-econômico, distribuição geográfica e de conhecimento. Revista Brasileira de Ciência e Movimento.2002:10(4):41-50.

[9] Craig CL, Marshall A, Sjöström M, Bauman AE, Booth $\mathrm{ML}$, Ainsworth BE, et al. International physical activity questionnaire: 12-country reliability and validity. Med Sci Sports Exerc. 2003;35(8):1381-95.

[10] Food and Agriculture Organization. The State of world fisheries and aquaculture (SOFIA) 2008: 2008. Rome, 2009. $196 \mathrm{p}$.

[11] Guedes AEL, Lyra CO, Cunha MV, Bandeira AVM, Henriques VMC, Soares FB, et al. Em torno da mesa: alimentando sensibilidades e competências: relato de uma experiência educativa em Macau, RN, Brasil. Seg Alim Nutr. 2008;15(1):1-14.

[12] Danelon MAS, Danelon MS, Silva MV. Serviços de alimentação destinados ao público escolar: análise da convivência do Programa de Alimentação Escolar e das cantinas. Seg Alim Nutr. 2006;13(1):85-94.

[13] Instituto Brasileiro de Geografia e Estatística. Pesquisa de Orçamentos Familiares - POF 2002-2003: microdados [acesso em 26 ago 2012]. Disponível em:http://www.ibge.gov.br/home/estatistica/populacao/c ondicaodevida/pof/2002analise/microdados.shtm

[14] Instituto Brasileiro de Geografia e Estatística. Pesquisa de Orçamentos Familiares - POF 2008-2009: microdados [acesso em 26 ago 2012]. Disponível em: http://www.ibge.gov.br/home/estatistica/populacao/condi caodevida/pof/2008 2009/microdados.shtm
[15] Wiefels R, Pereira G, Marquez Escudero H, Ayala M. Present and future markets for fish and fish products from small scale fisheries in Latin America: with special attention to the cases of México, Peru and Brazil. Rome: FAO, INFOPESCA, 2005. 33 p. [cited 2010 out 06]. Available from:http://www.infopesca.org/Downloads/publicaciones libre acceso/art13.pdf

[16] Brasil. Ministério da Pesca e Aquicultura (MPA). Campanha vai ressaltar os benefícios do consumo de pescado e o seu preparo. Brasília: MPA; 2009 [acesso em 26 ago 2012]. Disponível em: http://www.mpa.gov.br/mpa/seap/html/Comercializacao $\angle$ semana do peixe int $2009 . \mathrm{html}$

[17] Brasil. Ministério da Pesca e Aquicultura - MPA (2010) Boletim Estatístico da Pesca e Aquicultura 2008-2009. [acesso em 26 ago 2012]. Disponível em: http://www.sepaq.pa.gov.br/files/u1/anuario da pesca co mpleto.pdf

[18] Birgisdottir BE, Kiely M, Martinez JA, Thorsdottir I. Validity of a food frequency questionnaire to assess intake of seafood in adults in three European countries. Food Control. 2008;19(7):648-53.

[19] Mathias J, Baez JR. Análise setorial: a indústria do pescado. São Paulo: Panorama Setorial/Gazeta Mercantil, Legislação e estatísticas; 2003.

[20] Food and Agriculture Organization. Food Outlook.: global market analysis. Meat and meat products [cited 2011 nov 21]. Available from: http://www.fao.org/docrep/012/ak341e/ak341e09.htm

[21] Instituto de Economia Agrícola (IEA). Preços médios mensais no varejo [acesso em 21 nov 2011]. Disponível em: http://www.iea.sp.gov.br/out/index.php

[22] Companhia de Entrepostos e Armazéns Gerais de São Paulo (CEAGESP). Preços no atacado [acesso em 21 nov 2011]. Disponível em: http://www.ceagesp.gov.br/cotacoes

[23] Uilde AG, Farah TG, Flávia MM. Caracterização do consumo de carnes no Brasil. Revista Nacional da Carne. 2002;310:1-12.

[24] Baldini M, Pasqui F, Bordoni A, Maranesi M. Is the Mediterraneanlifestyle still a reality? Evaluation of food consumption and energy expenditure in Italian and Spanish university students. Public Health Nutr. 2009;12(2):148-55.

[25] Sonati J, Vilarta R. Novos padrões alimentares e as relações com os domínios da qualidade de vida e saúde. In:Vilarta R. (Org.). Novos padrões alimentares e as relações 
com os domínios da qualidade de vida e saúde. Campinas: IPES; 2010. p. 85-91.

[26] Munõz MA, Fíto M, Marrugat J, Covas MI, Schröder $\mathrm{H}$, et al. Adherence to the Mediterranean diet is associated with better mental and physical health. Br J Nutr. 2009:101(12),1821-27.

[27] Tyrovolas S, Panagiotakos DB. The role of Mediterranean type of diet on the development of cancer and cardiovascular disease, in the elderly: a systematic review. Maturitas. 2010;65(2):122-30.

[28] Maciel ES, Oetterer M. O desafio da alimentação como fator de qualidade de vida na última década. In: Vilarta $R$. (Org.). Novos padrões alimentares e as relações com os domínios da qualidade de vida e saúde. Campinas: IPES; 2010. p. 18-26.

[29] Engel JF, Blackwell RD, Miniard PW. Consumer behavior. 6o ed. Forth Worth: The Dryden Press; 1990.
[30] Arima HK. Consumo dos diferentes tipos de carne ainda é uma questão de preço. Situação européia. TecnoCarnes. 1996;6(3):1.

[31] Souki GQ, Salazar GT, Antonialli LM, Pereira CA. Atributos que afetam a decisão de compra dos consumidores de carne bovina. OR \& A. Revista de Administração da UFLA. 2003;5(2):36-51.

[32] Kubitza F. Com a palavra os consumidores. Revista Panorama da Aquicultura. 2002;69:48-53.

[33] Hobbs JE. Consumer demand for traceability. In: International agricultural Trade Research Consortium Annual Meeting, 2002, Monterey, CA. Saskatoon: Department of Agricultural Economics, University of Saskachewan, 2003. Working Paper, 3. 